\title{
Odorants and Their Contributions to Overall Odour Emission from a Landfill Leachate
}

\author{
Xinguang Wang, Gavin Parcsi, Eric Sivret, Minh Le, Richard Stuetz \\ Water Research Centre, University of New South Wales, Sydney 2052, Australia \\ Corresponding: xwang185@hotmail.com
}

\begin{abstract}
As one of the important odour sources, landfill sites have drawn more and more public attentions. Odour emissions from landfill sites depend on the waste buried, operation activities, running conditions, etc. A study for finding out all possible odorous compounds from a landfill was conducted by analysing of on-site gas phase samples and emission samples from a landfill leachate in Sydney, Australia using thermal desorber - gas chromatography - mass spectrometer (TD-GC-MS) and air server - thermal desorber - gas chromatography sulfur chemiluminoscence detector (AS-TD-GC-SCD). 49 odorants were identified from emission gas samples collected from landfill leachate collection pipe and only 8 odorants were detected from flux hood emission samples of the collected leachate sample. This indicates that more sampling and measurement techniques are always better to acquire all possible pollutants from an unknown odour source. The contributions of these odorants to overall odour emissions were also calculated based on their concentrations and odour thresholds. The top 10 odorants from leachate transportation pipe include methyl mercaptan, ethyl mercaptan, m-xylene, $\mathrm{H}_{2} \mathrm{~S}, \mathrm{CS}_{2}, 1,2,3,4$-tetra-methylbenzene, p-xylene, 1,2,4-trimethylbenzene, ethylbenzene and $\alpha$-pinene. They contributed more than $95 \%$ to the odour in the gas accumulated in the leachate collection pipe.
\end{abstract}

Keywords: odour, odorants, emission, landfill, leachate

\section{Introduction}

Landfill is still the predominant disposal method to dispose solid industrial and municipal waste in most countries. After moving to a landfill site, piled and buried waste will emit landfill gases because of chemical and biodegradation of the waste under anaerobic condition. Besides greenhouse gases like $\mathrm{CH}_{4}$ and $\mathrm{CO}_{2}$, the toxic and health issues of the minor gas components such as volatile organic compounds (VOCs), volatile sulfur compounds (VSCs), etc. have drawn more and more public attentions. Odour also becomes an important issue as more and more complaints raised by residents living around the landfill sites.

Since 1980s, many researchers have tried to specify and quantify composition of emission gases from various landfill sites for disposal of municipal, industrial, construction and demolition, or mixture of different wastes. Table 1 summarised the key investigations that have been done in past 3 decades. It can be seen from these publications that:

(1) Some of the studies focused only on few specific compounds like reduced sulfur compounds (RSCs): e.g. [1-4], and most of other works paid more attentions on more complicate VOCs such as [5-8], etc.

(2) Investigation sample sources include ambient air, waste gas at different depths, vent well, emissions from cover soil, leachate, etc. Most of samples were collected from the ambient air above the landfill sites or at the boundaries. For ambient air sampling, different sampling heights had been used: 1m [2], 1.5m [9-10], 2m [11], or several different heights [12]. In some studies, even no specification can be found in some of their publications.

(3) Limited gas emission data had reported from the cover soil, landfill waste using dynamic flux hood [13], static flux chamber [4], flux box [14], unspecified hood [5], or wind tunnel [7].

(4) Sampling method varied from researchers to researchers. Generally, gas phase sampling was conducted by using canister [15-16], single or multi-bed sorbent tubes, sampling bags, and liquid trapping.

Liquid trapping was used mainly for $\mathrm{NH}_{3}$ and $\mathrm{H}_{2} \mathrm{~S}$ spectrophotometer analysis [17-19]. [20] also used liquidtrapping for sampling of VFAs. Several sorbent tubes had been used: activated carbon or synthetic carbon+silic gel [17, 21-22], Tenax or Tenax GR [9, 17, 23], Porapak Q [17, 24], Carbotrap 3000 [11], and multi-bed sorbent $[5,13-14,25-27]$.

Sampling bags were mainly Tedlar [1-3, 20, 24, 28-32], Nalophan [5, 7-8, 33-34], bioriented polyester [10], or plastic [4] bags.

Other sampling methods used in other studies include diffusive sampler [34], passive sampling [35], and direct sampling to the $\mu \mathrm{GC}-\mathrm{MS}$ in the field [35].

(5) Component separation is almost all done by using gas chromatography (GC) for analysis of VOC and other compounds. However analytical method differed mainly by used sample processing procedures and detectors. 
Table 1 Compounds identified from landfill sites

\begin{tabular}{|c|c|c|c|c|c|}
\hline Compounds & Sample Collected & Sources & Sampling Method & Analytical Method & Reference \\
\hline $7 \mathrm{VOCs}+\mathrm{DMDS}$ & Diffusive samples & Solid waste treatment plant & Diffusive samplers & TD-GC-MS & {$[34]$} \\
\hline $\begin{array}{l}\mathrm{CH}_{2} \mathrm{Cl}_{2}, \mathrm{C}_{2} \mathrm{H}_{2} \mathrm{Cl}_{2}, \mathrm{C}_{2} \mathrm{HCl}_{3}, \\
\mathrm{C}_{2} \mathrm{Cl}_{4}, \mathrm{H}_{2} \mathrm{~S}, \mathrm{CH}_{3} \mathrm{SH}\end{array}$ & Boundaries, 500m away & Landfill sites & $\begin{array}{l}\text { Tenax tubes } \\
\text { Liquid trapping }\end{array}$ & $\begin{array}{l}\text { TD-GC } \\
\text { Spectrophotometer }\end{array}$ & {$[18]$} \\
\hline $68 \mathrm{VOCs}, \mathrm{NH}_{3}, \mathrm{H}_{2} \mathrm{~S}$ & Ambient air & Landfill & Nalophan bags + SPME & GC-MS & [19] \\
\hline $22 \mathrm{VOCs}$ & Ambient air (1.5m) & Solid waste treatment plant & Tenax GR tubes & TD-GC-MS & [9] \\
\hline 53 VOCs + 10 VFAs & Soil, water surface (hood) & Landfill & $\begin{array}{l}\text { Nalophan }+ \text { Tenax TA \& } \\
\text { Carboxen } 1000 \text { tubes }\end{array}$ & TD-GC-MS & {$[5]$} \\
\hline $16 \mathrm{VOCs}$ & Ambient air & MSW & Activated carbon tubes & CS2 extraction/GC-MS & {$[21]$} \\
\hline $\begin{array}{l}\text { More than } 80 \text { siloxanes }+ \\
\text { VOCs }\end{array}$ & Biogas & $\begin{array}{l}\text { Domestic waste disposal } \\
\text { sites }\end{array}$ & Canisters & TD-GC-MS/AED & {$[36]$} \\
\hline $\begin{array}{l}\text { Benzene, VCM, PCDD/Fs, } \\
\text { DL-PCBs \& PAHs }\end{array}$ & $\begin{array}{l}\text { Diffusive surface, point } \\
\text { emission }\end{array}$ & MSW landfill & Nelophan bags + SPME & GC-MS & {$[33]$} \\
\hline 19 VOCs & Air & $\begin{array}{l}\text { Municipal waste organic } \\
\text { fraction treatment plant }\end{array}$ & $\begin{array}{l}\text { Synthetic carbon }+ \text { silica gel } \\
\text { tubes }\end{array}$ & $\begin{array}{l}\text { CS2 extraction/GC-MS/HPLC- } \\
\text { UV }\end{array}$ & {$[22]$} \\
\hline $63 \mathrm{VOCs}$ & Surface (flux box) & MSW disposal sites & $\begin{array}{l}\text { Chromosorb } 106 \& \text { \& } \\
\text { Carbopack cartridges }\end{array}$ & TD-GC-MS & {$[14]$} \\
\hline 41-66 trace compounds & Ambient air $(1.5 \mathrm{~m})$ & $\begin{array}{l}\text { Landfill for MSW + } \\
\text { composting residue }\end{array}$ & Bioriented polyester bags & Pre-concentrated/GC-MS & {$[10]$} \\
\hline 38-66 VOCs & Ambient air $(2 \mathrm{~m})$ & MSW & Carbotrap 300 tubes & $\begin{array}{l}\text { Aerotrapt purge and trap } \\
\text { concentrator-GC-MS }\end{array}$ & [11] \\
\hline $\mathrm{H}_{2} \mathrm{~S}+$ over 100 VOCs & Waste gas (1.0-4.3m deep) & $\begin{array}{l}\text { Domestic and industrial } \\
\text { waste landfill }\end{array}$ & Tenax or Porapak Q tubes & $\begin{array}{l}\text { GC-MS } \\
\text { Spectrophotometer }\end{array}$ & {$[17]$} \\
\hline VOCs & $\begin{array}{l}5 \text { different height of open } \\
\text { cells }\end{array}$ & MSW & Activated carbon tubes & $\mathrm{CS}_{2}$ extraction to GC-MS & {$[12]$} \\
\hline 140 VOCs & Ambient air & Landfill & Nalophan bags & USEPA TO-15 & [7] \\
\hline $\begin{array}{l}\text { Hundreds VOCs with } 7 \\
\text { presenting }\end{array}$ & Ambient air & Landfill area & $\begin{array}{l}\text { Tenax \& Spherocarb } \\
\text { cartridge }\end{array}$ & TD-GC-MS & {$[23]$} \\
\hline 48-66 VOCs & Soil \& well gas & Landfill & Glass tubes & SPME/GC-MS & [6] \\
\hline $\begin{array}{l}93 \text { VOCs with } 70 \text { positively } \\
\text { identified }\end{array}$ & Ambient air & MSW treatment plants & Tenax TA \& Carboxen 1000 & TD-GC-MS & [27] \\
\hline 147 VOCs & Laboratory reactor & Real waste sample & Nahophan bag & SPME/GC-MS & [8] \\
\hline Over 500 compounds & Air & Municipal landfill & $\begin{array}{l}\text { Passive sampling } \\
\text { Direct sampling }\end{array}$ & $\begin{array}{l}\text { GC-MS } \\
\mu \mathrm{GC}-\mathrm{MS}\end{array}$ & {$[35]$} \\
\hline $100 \mathrm{VOCs}$ & Ambient air & Landfill & Nalophan bags & SPME/GC-MS & [32] \\
\hline $60 \mathrm{VOCs}$ & Closed surface (flux hood) & Landfill & Multi-bed sorbent tubes & TD-GC-MS & [13] \\
\hline
\end{tabular}




\begin{tabular}{|c|c|c|c|c|c|}
\hline $\mathrm{H}_{2} \mathrm{~S} \&$ VOCs & $\begin{array}{l}\text { Passive vent, landfill } \\
\text { cover, leachate }\end{array}$ & Landfill & Canister \& Tedlar bags & $\begin{array}{l}\text { GC/FID/PID/ELCD } \\
\text { Jerome }\end{array}$ & {$[15]$} \\
\hline 12 sulfur compounds & Duct & Domestic landfill & Tedlar bag+Porapak Q tube & TD-GC-MS & [24] \\
\hline 3 sulfur compounds & Flux hood sample & Landfill & $\begin{array}{l}\text { Glass fiber filters } \\
\text { impregnated with mercuric } \\
\text { acetate }\end{array}$ & GC-FID/SCD & [37] \\
\hline $\mathrm{H}_{2} \mathrm{~S}$ & Cover soil & $\begin{array}{l}\text { Construction \& demolition } \\
\text { debris landfill }\end{array}$ & Tedlar bags & Jerome & [31] \\
\hline $\begin{array}{l}\text { RSCs, carbonyls, } \mathrm{NH}_{3} \text {, } \\
\text { VOCs, VFAs }\end{array}$ & Vent & $\begin{array}{l}\text { Landfill leachate treatment } \\
\text { plant }\end{array}$ & $\begin{array}{l}\text { Tedlar bags } \\
\text { DNPH cartridge } \\
\text { Liquid trapping } \\
\text { Tedlar bag } \\
\text { Liquid trapping } \\
\end{array}$ & $\begin{array}{l}\text { TD-GC-MS } \\
\text { HPLC-UV } \\
\text { UV/VIS } \\
\text { TD-GC-MS/FID } \\
\text { SPME/TD-GC-FID } \\
\end{array}$ & [20] \\
\hline $\begin{array}{l}\mathrm{NH}_{3} \text {, aromatics, sulfur } \\
\text { compounds, oxygenated } \\
\text { compounds, amines, fatty } \\
\text { acid }\end{array}$ & Vent system & Landfill & Tedlar bags & $\begin{array}{l}\text { SPME/GC-FID, GC-PFPD, } \\
\text { HPLC, etc. }\end{array}$ & [30] \\
\hline 5 sulfur compounds & $\begin{array}{l}\text { Active, inactive, covered } \\
\text { area }\end{array}$ & Landfill & Plastic bags & USEPA TO-15 & {$[4]$} \\
\hline 5 sulfur compounds & Ambient air & Landfill & Tedlar bags & TD-GC-PFPD & [29] \\
\hline Sulfur compounds & $\begin{array}{l}\text { Landfill gas, soil vapore, } \\
\text { ambient air }\end{array}$ & Landfill & Tedlar bags & $\begin{array}{l}\text { Jerome, purge \& trap } \\
\text { concentrator/GC-MS }\end{array}$ & [3] \\
\hline $5 \mathrm{RSCs}$ & Vent pipes & MSW & Tedlar bags & TD-GC-PFPD & {$[1]$} \\
\hline $5 \mathrm{RSCs}$ & Vent pipes & 4 landfill sites & Tedlar bags & TD-GC-PFPD & {$[28]$} \\
\hline $4 \mathrm{RSCs}$ & Ambient air $(1 \mathrm{~m})$ & 2 landfill sites & Tedlar bags & TD-GC-PFPD & {$[2]$} \\
\hline VOCs & Leachate & Industrial landfill & SPE/SPME & $\begin{array}{l}\text { GC-MS, LC-MS, NMR, LC- } \\
\text { NMR }\end{array}$ & [38] \\
\hline $\begin{array}{l}\text { Over } 200 \text { compounds with } \\
35 \text { priority pollutants }\end{array}$ & Leachate & MSW & Multi-bed carbotrap tubes & TD-GC-MS & {$[26]$} \\
\hline $\begin{array}{l}\text { VOCs + inorganic } \\
\text { compounds }\end{array}$ & $\begin{array}{l}\text { Leachate + suspended } \\
\text { particles }\end{array}$ & Landfill sites & Solvent extraction & HPLC/GC-MS/FID & [39] \\
\hline 180 VOCs (22 quantified) & Seepage, leakage & Landfill leachate & Solvent extraction & $\begin{array}{l}\text { GC-FID/ECD } \\
\text { GC-MS }\end{array}$ & [36] \\
\hline 11 predominant VOCs & Leachate & Landfill & Liquid-liquid extraction & GC-MS & {$[40]$} \\
\hline 45 organic compounds & Leachate & Municipal landfill & Solvent extraction & GC-MS & {$[41]$} \\
\hline
\end{tabular}


The most often used detector for VOC analysis is mass spectrometer (MS), which made GC-MS the basic configuration for VOC analysis. Pulsed flame photometric detector (PFPD) was used for analysis of sulfur compounds [1-2, 28-29]. Other detectors include FID (flame ionization detector), PID (photoionisation detector), ELCD (electrolytic conductivity detector), ECD (electron capture detector), AED (atomic emission detector), SCD (sulfur chemiluminoscence detector), UV (UV detector) were also used in some studies.

In these studies, most of the sample processing procedures utilised thermal desorber (TD) when canisters, bags, or sorbent tubes samples were collected. So, the most frequently used instrument combination is TD-GCMS [5, 9, 13-14, 16, 23-25, 27, 34]. In some cases when bag samples were collected, solid phase microextraction (SPME) technique was employed for GC-MS sample processing [6, 8, 19, 30, 32-33]. [20] also used SPME to absorb VFAs from head space of liquid-trapping samples.

(6) $\mathrm{H}_{2} \mathrm{~S}$ was also measured directly or indirectly (sampled to the bag before the measurement) by using Jerome gold film analyzer $[3,15,31]$ in the field.

(7) No leachate emission data had reported although leachate composition was reported after HPLC/GCMS/FID analysis [39], SPE/SPME/GC-MS, LC-MS, NMR, LC-NMR analysis [38], or GC-MS analysis after liquid-liquid extraction [36, 40-41].

(8) VOCs and sulfur compounds were two major groups of compounds investigated. The number of detected compounds varied quite significantly from one publication to another not just because of the differences between landfill sites, but also because of what the investigators focused on, and the sampling and analytical techniques available for using during their investigation.

(9) Odorous compounds were specially spotted in some of the studies. [42] summarised the top 15 odorous compounds present in landfill gas (Table 2). $\mathrm{NH}_{3}$ was measured by some researchers [19, 43]. As odorous compounds, $\mathrm{H}_{2} \mathrm{~S}$ and/or up to 14 other sulfur compounds from landfill sites were reported by [3, 8, 17, 19, 24, 28-29, 31, 34, 37, 43-44]. Apart from 7 sulfides (DMDS, $\mathrm{CS}_{2}$, methyl propyl disulfide, allyl mercaptan, DMTS, allyl methyl sulfide, 1-propene-1-methylthio), 2 nitrides (trimethylamine and 2-methyl pyridine) were also thought to be the main odour emission contributors [8].

Some of the VOCs and VFAs were also thought to cause the annoying odour at the landfill sites: alkylbenzene, terpenes and limonene [11]; 2-butanone, $\alpha$-pinene, tetrachloroethylenen, $\beta$-pinene, limonene, phenol and benzoic acid [34]; acetic acid, 2,3-butanedione, ethyl acetate, $\alpha$-pinene, and limonene [9]; acetic acid and n-butyraldehyde [30]], methyl butyrate, ethyl butyrate,propionate, butyric acid, limonene, and butan-2-ol [44]; esters and ethyl butanoate [17]; aldehydes, ketones, and esters [5]; toluene, styrene, xylene, methyl ethyl ketone, methyl isobutyl ketone, butyl acetate, isobutyl alcohol, propionic acid, butyric acid, isovaleric acid, and valeric acid [20].

Table 2 Most odorous compounds present in landfill gas [42]

\begin{tabular}{cll}
\hline Rank Odour Importance & \multicolumn{1}{c}{ by Hurst et al 2005 } & \multicolumn{1}{c}{ by Parker et al 2002 } \\
\hline 1 & Methanethiol & Hydrogen sulfide \\
2 & Ethyl butanoate & Methanethiol \\
3 & Ethanethiol & Butanoic acid \\
4 & Dimethyl sulfide & Ethanal \\
5 & Dimethyl disulfide & Carbon disulfide \\
6 & Diethyl sulfide & Ethyl butanoate \\
7 & Butanoic acid & 1-propanethiol \\
8 & Hydrogen sulfide & Dimethyl disulfide \\
9 & 1,1-dichloroethane & Ethanethiol \\
10 & Diethyl ether & 1-pentene \\
11 & Propan-1-ol & Dimethyl sulfide \\
12 & Methyl butanoate & 1-butanethiol \\
13 & Pentanethiols & Propanoic acid \\
14 & Propyl propanoate & Butyl ethanoate \\
15 & Propyl benzenes & Butanal \\
\hline
\end{tabular}

(10) From the leachate, only [26] specified odour contributors that included aromatic hydrocarbons, ketones, alcohols, sulfur containing compounds, pyridines, pyrizins, carvone, cyclic esters, and dioxolans. In this study, odorants emitted from the leachate of a municipal waste landfill site were investigated. The emission gas from the leachate transport pipe and emission gas from the leachate surface were sampled for TDGC-MS and air server-TD-GC-SCD analysis. Based on the identified compounds, and their odour threshold (OT) values, contributions of the key odorants were calculated and ranked.

\section{Methodology}




\subsection{Sample collection}

Field sampling activities included fugitive gas emission samples collected from the liquid aeration return pipe stretching out above the leachate dam; and liquid sample collected from the leachate storage dam.

2.1.1 Fugitive gas sampling

Fugitive gas samples were collected for VOC and VSC analysis. The samples were collected from the leachate aeration liquid nozzle of the liquid aeration return pipe stretching out above the leachate dam.

VOC samples were collected on-site using reconditioned Tenax TA sorbent tubes (Marks International). These samples were collected near the nozzle of the liquid aeration return pipe stretching out above the leachate dam. Calibrated sampling pumps (SKC AirChek 2000) were used to draw the gas phase stream through the sorbent tubes (collection rate $100 \mathrm{~mL} / \mathrm{min}$; duration $10 \mathrm{~min}$; sample volume $1 \mathrm{~L}$ ). VOC samples were collected in duplicates, each of which had two tubes connected in series to confirm if there is any potential breakthrough of analytes absorbed to the first sampling tube.

VSC samples were collected in duplicate into $5 \mathrm{~L}$ Tedlar ${ }^{\mathrm{TM}}$ bags utilising a lung sampler (collection rate 2.5 $\mathrm{L} / \mathrm{min}$ ).

\subsubsection{Leachate and its emission sampling}

A $5 \mathrm{~L}$ composite leachate sample was collected from the leachate storage dam into an amber glass bottle with no headspace. The sample bottle was kept refrigerated before the emission test. It was used to collect emission samples as the leachate dam was not safely accessible for emission sampling using flux hood chamber [48] or portable wind tunnel [49] surface source sampling device.

The emission simulation test of the leachate was conducted using a flux hood chamber in compliance with Australian Standard method [50]. This method was also approved by the Department of Environment and Conservation for sampling emissions from a surface area [51]. Prior to sampling, the flux hood chamber was seated on the leachate surface and purged with high purity nitrogen gas for $30 \mathrm{~min}$ at the flow rate of $5 \mathrm{~L} / \mathrm{min}$ before the sampling. Duplicate bag samples $\left(5 \mathrm{~L}\right.$ Tedlar $\left.{ }^{\mathrm{TM}}\right)$ were collected using a lung sampler (collection rate $2.5 \mathrm{~L} / \mathrm{min}$ ) for VSC analysis. Duplicate Tenax TA sorbent tube samples were also collected (collection rate 100 $\mathrm{mL} / \mathrm{min}$ for duration of $30 \mathrm{~min}$ with total sampling volume of $3 \mathrm{~L}$ ) for VOC analysis.

\subsection{Analyses of VOCs and VSCs}

Sorbent tube analysis was performed using an Ultra autosampler equipped thermal desorber (TD, Ultra + Unity II Markes International, UK) - gas chromatograph - mass spectrometer (GC-MS, 7890A GC + 5975C Agilent Technologies) system. The thermal desorber was fitted with a general purpose graphitised carbon analyte focussing trap (U-T11GPC-2S, Markes International UK); the gas chromatograph was fitted with a low polarity DB-VRX (Agilent Technologies) capillary column $(30 \mathrm{~m} \times 0.25 \mathrm{~mm} \times 1.4 \mu \mathrm{m})$ for analyte separation; GC carrier gas was ultra high purity helium $(1.8 \mathrm{~mL} / \mathrm{min})$. GC oven temperature was programmed initially setup at $50^{\circ} \mathrm{C}$ for 2 min then increased to $200^{\circ} \mathrm{C}$ with an increment of $15^{\circ} \mathrm{C} / \mathrm{min}$ and held for $3 \mathrm{~min}$. Analyte speciation was performed by the mass selective detector operating in continuous scan mode $(35-335 \mathrm{~m} / \mathrm{z})$; NIST02 and NIST11 libraries were used for spectra matching and compound identification. Gas phase TO-17 standard (from Air Liquid) was used for calibration and quantification of some compounds, and all other compounds were quantified based on their peak area against toluene calibration factor.

VSC bag samples were analysed on the same day of the field sampling. Sample bags were directly sampled by an air server (AS, Markes International) coupled to a thermal desorber (TD) Unity (Markes International) and analysed by gas chromatograph (GC, Agilent Technologies) coupled to a sulfur chemiluminoscence detector (SCD, Agilent Technologies). The thermal desorber was fitted with a sulfur specific carbon molecular sieve analyte focussing trap (U-T6SUL-2S, Markes International UK). A DB-VRX column $(30 \mathrm{~m} \times 0.25 \mathrm{~mm} \times 1.4 \mu \mathrm{m})$ was used for GC separation, initially starting at $37^{\circ} \mathrm{C}$ held for 3 minutes; the temperature was increased at $15^{\circ} \mathrm{C} / \mathrm{min}$ up to $225^{\circ} \mathrm{C}$ and held for 2 minutes. The flow rate of the carrier gas (ultrahigh purity helium) was set as $1.5 \mathrm{~mL} / \mathrm{min}$. The sulfur compounds determined by TD-GC-SCD system include carbonyl sulfide, hydrogen sulfide, merthyl mercaptan, ethyl mercaptan, dimethyl sulfide, carbon disulfide, 2-methyl-2-propantiol, ethyl methyl sulfide, 2-butanehiol, 1-butanethiol, dimethyl disulfide, ethyl methyl sulfide, diethyl disulfide, and dimethyl trisulfide. Their quantifications were based on high-purity individual liquid standards purchased from Sigma-Aldrich, Arkema Inc. or SAFC Supply Solutions using the dynamic injection sample preparation method [52].

\subsection{Calculation of odour emission contribution}

Based on the measurements, odour unit factor was calculated for all quantifiable components using the equation (1)

$$
O U F_{i}=C_{i} / O T_{i}
$$


where $O U F_{i}$ is the odour unit factor of an odourant; $C_{i}$ is concentration $\left(\mathrm{mg} / \mathrm{m}^{3}\right) ; O T_{i}$ is the odour threshold concentration $\left(\mathrm{mg} / \mathrm{m}^{3}\right)$.

Contribution of an odorant to total odours can be calculated as

$$
c_{i}=O U F_{i} / \sum O U F_{i} \cdot 100 \%
$$

where $c_{i}$ is the contribution of an odorant to total odours.

\section{Results and Discussion}

\subsection{Identified odorants}

Over 130 VOCs and VSCs were detected from the gas samples collected from the leachate pipe; among which at least 49 of them are documented odorants (Table 3). These compounds are mainly hydrocarbons (29), sulfur compounds (15), halogenalkanes (2), and oxygenated hydrocarbons (3).

Table 4 lists odorants found in the flux hood emission samples of collected leachate sample. Comparing with the field gas sample results (Table 3), it can be seen that much less odorous compounds were identified. The reason for this may be: (1) some of odorants had mostly evaporated after the leachate was stored in the leachate dam for a period of time; (2) the leachate in the dam had been diluted by rain water; (3) most of odorants had low evaporation ability or they were trapped in the liquid phase when the emission test was carried out; (4) field gas sample may also include landfill gases that appear unusually in the leachate.

Almost all of these odorous VOCs were detected by many of previous researchers from the landfill gases although not all of them had been mentioned to be odorous.

In total, 17 sulfur compounds were detected form field gas samples, of which 14 VSCs (only 12 were listed in Table 3 because ethyl methyl disulfide and diethyl disulfide had not been quantified as they only showed small peaks) were detected by TD-GC-SCD method and 3 more (sulfur dioxide, 1-propanethiol and 2-propanethiol) were detected by TD-GC-MS.

Apart from the common sulfur compounds like $\mathrm{H}_{2} \mathrm{~S}$, methyl mercaptan, dimethyl sulfide (DMS), $\mathrm{CS}_{2}$, and dimethyl disulfide (DMDS) reported by other researches, this study also detected other VSCs with very limited reports such as cabonyl sulfide by [2, 24]; ethyl mercaptan, 2-methyl-2-propanthiol, 2-butanethiol by [2]; 2butanethiol by [2, 27]; 1-butanethiol by [24, 42]; DES by [4]; DMTS by [8, 30]. Ethyl methyl disulfide could be firstly detected from landfill as it was not on the listed sulfur compounds detected in previous studies. This may be because GC-SCD is the extremely powerful tool to characterise and quantify a wide range of low-level sulfur species in gaseous streams [53]

Table 3 Odorants identified in gas phase field samples

\begin{tabular}{|c|c|c|c|c|c|c|c|c|}
\hline \multirow{2}{*}{$\underset{\mathbf{k}}{\operatorname{Ran}}$} & \multirow{2}{*}{ Compound } & \multirow{2}{*}{$\begin{array}{c}\text { Formul } \\
\mathbf{a}\end{array}$} & \multirow{2}{*}{ MW } & \multirow{2}{*}{$\begin{array}{c}\text { Odour } \\
\text { Descripto } \\
\mathbf{r}\end{array}$} & \multicolumn{2}{|c|}{ OT } & \multirow{2}{*}{$\begin{array}{c}\text { Concentratio } \\
\text { n }\left(\mathbf{m g} / \mathbf{m}^{3}\right)\end{array}$} & \multirow{2}{*}{$\begin{array}{c}\text { Odour } \\
\text { Contributio } \\
\text { n }(\%)\end{array}$} \\
\hline & & & & & $\mathrm{ppm}^{[45]}$ & $\underset{]}{\mathrm{mg} / \mathrm{m}^{3[46}}$ & & \\
\hline 1 & $\begin{array}{c}\text { Methyl } \\
\text { mercaptan }\end{array}$ & $\mathrm{CH}_{3} \mathrm{SH}$ & 48.11 & $\begin{array}{l}\text { Rotton } \\
\text { cabbage }\end{array}$ & 0.00007 & & 0.0156 & 29.29 \\
\hline 2 & $\begin{array}{c}\text { Ethyl } \\
\text { mercaptan }\end{array}$ & $\mathrm{C}_{2} \mathrm{H}_{5} \mathrm{SH}$ & 62.13 & $\begin{array}{l}\text { Leek, onion } \\
\text { odour }\end{array}$ & $\begin{array}{c}0.000008 \\
7\end{array}$ & & 0.0013 & 15.19 \\
\hline 3 & $m$-xylene & $\mathrm{C}_{8} \mathrm{H}_{10}$ & $\begin{array}{c}106.1 \\
7\end{array}$ & $\begin{array}{l}\text { Sweet, } \\
\text { aromatic }\end{array}$ & 0.041 & & 8.8859 & 12.89 \\
\hline 4 & $\begin{array}{l}\text { Hydrogen } \\
\text { sulfide }\end{array}$ & $\mathrm{H}_{2} \mathrm{~S}$ & 34.08 & Rotten egg & 0.00041 & & 0.0257 & 11.61 \\
\hline 5 & $\begin{array}{l}\text { Carbon } \\
\text { disulfide }\end{array}$ & $\mathrm{CS}_{2}$ & 76.14 & $\begin{array}{l}\text { Sweetish } \\
\text { aromatic }\end{array}$ & 0.21 & & 24.601 & 9.72 \\
\hline 6 & $\begin{array}{c}\text { 1,2,3,4-tetra- } \\
\text { methyl- } \\
\text { benzene }\end{array}$ & $\mathrm{C}_{10} \mathrm{H}_{14}$ & $\begin{array}{c}134.2 \\
2\end{array}$ & Aromatic & 0.0011 & & 0.1272 & 5.44 \\
\hline
\end{tabular}




\begin{tabular}{|c|c|c|c|c|c|c|c|}
\hline 7 & $p$-xylene & $\mathrm{C}_{8} \mathrm{H}_{10}$ & $\begin{array}{c}106.1 \\
7\end{array}$ & Sweet & 0.058 & 4.0263 & 4.13 \\
\hline 8 & $\begin{array}{c}\text { 1,2,4-trimetyl } \\
\text { benzene }\end{array}$ & $\mathrm{C}_{9} \mathrm{H}_{12}$ & $\begin{array}{c}120.1 \\
9\end{array}$ & Aromatic & 0.12 & 7.3886 & 3.23 \\
\hline 9 & Ethylbenzene & $\mathrm{C}_{8} \mathrm{H}_{10}$ & $\begin{array}{c}106.1 \\
7\end{array}$ & $\begin{array}{c}\text { Aromatic, } \\
\text { gasoline } \\
\text { type }\end{array}$ & 0.17 & 6.0946 & 2.13 \\
\hline 10 & $\alpha$-pinene & $\mathrm{C}_{10} \mathrm{H}_{16}$ & $\begin{array}{c}136.2 \\
4\end{array}$ & $\begin{array}{l}\text { Herbal, } \\
\text { terpenic }\end{array}$ & 0.018 & 0.807 & 2.08 \\
\hline 11 & $\begin{array}{l}\text { Carbonyl } \\
\text { sulfide }\end{array}$ & $\mathrm{COS}$ & 60.08 & $\begin{array}{c}\text { Distinct } \\
\text { sulfide } \\
\text { smell }\end{array}$ & 0.055 & 0.6884 & 1.32 \\
\hline 12 & Toluene & $\mathrm{C}_{7} \mathrm{H}_{8}$ & 92.14 & $\begin{array}{c}\text { Sweet, } \\
\text { pungent }\end{array}$ & 0.33 & 4.0797 & 0.85 \\
\hline 13 & $\begin{array}{c}\text { Methyl } \\
\text { cyclohexane }\end{array}$ & $\mathrm{C}_{7} \mathrm{H}_{14}$ & 98.19 & $\begin{array}{l}\text { Petroleum- } \\
\text { like }\end{array}$ & 0.15 & 1.377 & 0.59 \\
\hline 14 & Heptane & $\mathrm{C}_{7} \mathrm{H}_{16}$ & $\begin{array}{c}100.2 \\
0\end{array}$ & $\begin{array}{l}\text { Gasoline- } \\
\text { like }\end{array}$ & 0.67 & 2.9604 & 0.28 \\
\hline 15 & $\begin{array}{c}1,3,5- \\
\text { trimethyl- } \\
\text { benzene }\end{array}$ & $\mathrm{C}_{9} \mathrm{H}_{12}$ & $\begin{array}{c}120.1 \\
9\end{array}$ & $\begin{array}{l}\text { Distinctive, } \\
\text { aromatic }\end{array}$ & 0.17 & 0.8941 & 0.28 \\
\hline 16 & $\begin{array}{l}\text { 2-methyl- } \\
\text { heptane }\end{array}$ & $\mathrm{C}_{8} \mathrm{H}_{18}$ & $\begin{array}{c}114.2 \\
3\end{array}$ & $\begin{array}{l}\text { Natural gas } \\
\text { odour }\end{array}$ & 0.11 & 0.4849 & 0.24 \\
\hline 17 & Decane & $\mathrm{C}_{10} \mathrm{H}_{22}$ & $\begin{array}{c}142.2 \\
9\end{array}$ & $\begin{array}{c}\text { Petrolic } \\
\text { odour }\end{array}$ & 0.62 & 3.2479 & 0.23 \\
\hline 18 & Nonane & $\mathrm{C}_{9} \mathrm{H}_{20}$ & $\begin{array}{c}128.2 \\
6\end{array}$ & $\begin{array}{c}\text { Petrolic } \\
\text { odour }\end{array}$ & 2.2 & 6.1924 & 0.14 \\
\hline 19 & Octane & $\mathrm{C}_{8} \mathrm{H}_{18}$ & $\begin{array}{c}114.2 \\
3\end{array}$ & $\begin{array}{l}\text { Gasoline- } \\
\text { like }\end{array}$ & 1.7 & 2.1731 & 0.09 \\
\hline 20 & $\begin{array}{l}\text { 3-methyl- } \\
\text { hexane }\end{array}$ & $\mathrm{C}_{7} \mathrm{H}_{16}$ & $\begin{array}{c}100.2 \\
0\end{array}$ & $\begin{array}{l}\text { Petrolic } \\
\text { odour }\end{array}$ & 0.84 & 0.8938 & 0.07 \\
\hline 21 & Undecane & $\mathrm{C}_{11} \mathrm{H}_{24}$ & $\begin{array}{c}156.3 \\
1\end{array}$ & $\begin{array}{c}\text { Petrolic } \\
\text { odour }\end{array}$ & 0.87 & 1.3541 & 0.06 \\
\hline 22 & Dodecane & $\mathrm{C}_{12} \mathrm{H}_{26}$ & $\begin{array}{c}170.3 \\
4\end{array}$ & $\begin{array}{c}\text { Petrolic } \\
\text { odour }\end{array}$ & 0.11 & 0.1192 & 0.04 \\
\hline 23 & Hexane & $\mathrm{C}_{6} \mathrm{H}_{14}$ & 86.18 & $\begin{array}{l}\text { Petrolic, } \\
\text { gasoline- } \\
\text { like }\end{array}$ & 1.5 & 0.779 & 0.04 \\
\hline 24 & Benzene & $\mathrm{C}_{6} \mathrm{H}_{6}$ & 78.11 & $\begin{array}{l}\text { Sweet, } \\
\text { solvent }\end{array}$ & 2.7 & 0.9719 & 0.03 \\
\hline 25 & $\begin{array}{c}\text { Methyl- } \\
\text { cyclopentane }\end{array}$ & $\mathrm{C}_{6} \mathrm{H}_{12}$ & 84.16 & $\begin{array}{l}\text { Sweetish } \\
\text { gasoline- } \\
\text { line }\end{array}$ & 1.7 & 0.5073 & 0.02 \\
\hline 26 & $\begin{array}{l}\text { 3-methyl- } \\
\text { pentane }\end{array}$ & $\mathrm{C}_{7} \mathrm{H}_{16}$ & $\begin{array}{c}100.2 \\
0\end{array}$ & $\begin{array}{c}\text { Not } \\
\text { available }\end{array}$ & 0.37 & 0.0932 & 0.02 \\
\hline
\end{tabular}




\begin{tabular}{|c|c|c|c|c|c|c|c|c|}
\hline 27 & $\begin{array}{l}\text { 3-methyl- } \\
\text { heptane }\end{array}$ & $\mathrm{C}_{8} \mathrm{H}_{18}$ & $\begin{array}{c}114.2 \\
3\end{array}$ & $\begin{array}{l}\text { Petrolic } \\
\text { odour }\end{array}$ & 1.5 & & 0.4216 & 0.02 \\
\hline 28 & 1-pentane & $\mathrm{C}_{5} \mathrm{H}_{10}$ & 70.13 & $\begin{array}{l}\text { Gasoline- } \\
\text { like, } \\
\text { hydrocarbo } \\
\text { n odour }\end{array}$ & 0.1 & & 0.0097 & 0.01 \\
\hline 29 & $\begin{array}{l}\text { 2-methyl- } \\
\text { pentane }\end{array}$ & $\mathrm{C}_{6} \mathrm{H}_{14}$ & 86.18 & $\begin{array}{l}\text { Gasoline- } \\
\text { like }\end{array}$ & 7 & & 0.4770 & $<0.01$ \\
\hline 30 & $\begin{array}{c}\text { Sulfur } \\
\text { dioxide }\end{array}$ & $\mathrm{SO}_{2}$ & 64.07 & $\begin{array}{l}\text { Burnt } \\
\text { match }\end{array}$ & 0.87 & & 0.0314 & $<0.01$ \\
\hline 31 & $\begin{array}{l}\text { Dimethyl } \\
\text { sulfide }\end{array}$ & $\mathrm{C}_{2} \mathrm{H}_{6} \mathrm{~S}$ & 62.13 & $\begin{array}{l}\text { Cabbage, } \\
\text { sulfurous }\end{array}$ & 0.003 & & 0.0001 & $<0.01$ \\
\hline 32 & $\begin{array}{l}\text { 4-methyl- } \\
\text { heptane }\end{array}$ & $\mathrm{C}_{8} \mathrm{H}_{18}$ & $\begin{array}{c}114.2 \\
3\end{array}$ & $\begin{array}{l}\text { Gasoline- } \\
\text { like }\end{array}$ & 1.7 & & 0.0910 & $<0.01$ \\
\hline 33 & 4-octane & $\mathrm{C}_{8} \mathrm{H}_{16}$ & $\begin{array}{c}112.2 \\
1\end{array}$ & $\begin{array}{l}\text { Gasoline- } \\
\text { like }\end{array}$ & 1.7 & & 0.0672 & $<0.01$ \\
\hline 34 & $\begin{array}{l}\text { Dimethyl } \\
\text { disulfide }\end{array}$ & $\mathrm{C}_{2} \mathrm{H}_{6} \mathrm{~S}_{2}$ & 94.20 & $\begin{array}{l}\text { Cabbage, } \\
\text { sulfurous }\end{array}$ & 0.0022 & & 0.00007 & $<0.01$ \\
\hline 35 & $\begin{array}{l}\text { 3-methyl- } \\
\text { pentane }\end{array}$ & $\mathrm{C}_{6} \mathrm{H}_{14}$ & 86.18 & $\begin{array}{l}\text { Gasoline- } \\
\text { like }\end{array}$ & 8.9 & & 0.1851 & $<0.01$ \\
\hline 36 & $\begin{array}{c}\text { Tetra- } \\
\text { hydrofuran }\end{array}$ & $\mathrm{C}_{4} \mathrm{H}_{8} \mathrm{O}$ & 72.11 & Ether-like & $30^{[47]}$ & & 0.0061 & $<0.01$ \\
\hline 37 & $\begin{array}{l}\text { 2,2-dimethyl- } \\
\text { butane }\end{array}$ & $\mathrm{C}_{6} \mathrm{H}_{14}$ & 86.18 & $\begin{array}{l}\text { Gasoline- } \\
\text { like }\end{array}$ & 20 & & 0.0045 & $<0.01$ \\
\hline 38 & 2-butanethiol & $\mathrm{C}_{4} \mathrm{H}_{10} \mathrm{~S}$ & 90.19 & $\begin{array}{l}\text { Heavy } \\
\text { skunk, foul } \\
\text { sulfur }\end{array}$ & & 0.007 & 0.311636 & $<0.01$ \\
\hline 39 & $\begin{array}{l}\text { 2-methyl-2- } \\
\text { propanthiol }\end{array}$ & $\mathrm{C}_{4} \mathrm{H}_{10} \mathrm{~S}$ & 90.19 & Skunk & & 0.00009 & 0.001 & $<0.01$ \\
\hline 40 & $\begin{array}{c}2- \\
\text { propanethiol }\end{array}$ & $\mathrm{C}_{3} \mathrm{H}_{8} \mathrm{~S}$ & 76.16 & $\begin{array}{l}\text { Cabbage- } \\
\text { like }\end{array}$ & & 0.0081 & 0.0468 & $<0.01$ \\
\hline 41 & Butane & $\mathrm{C}_{4} \mathrm{H}_{10}$ & 58.12 & $\begin{array}{l}\text { Gasoline- } \\
\text { like }\end{array}$ & 1200 & & 0.0155 & $<0.01$ \\
\hline 42 & $\begin{array}{l}\text { Dimethyl } \\
\text { trisulfide }\end{array}$ & $\mathrm{C}_{2} \mathrm{H}_{6} \mathrm{~S}_{3}$ & $\begin{array}{c}126.2 \\
6\end{array}$ & $\begin{array}{l}\text { Rotten } \\
\text { cabbage }\end{array}$ & & 0.00006 & 0.00013 & $<0.01$ \\
\hline 43 & $\begin{array}{c}1- \\
\text { propanethiol }\end{array}$ & $\mathrm{C}_{3} \mathrm{H}_{8} \mathrm{~S}$ & 76.16 & $\begin{array}{l}\text { Cabbage- } \\
\text { like }\end{array}$ & & 0.018 & 0.0233 & $<0.01$ \\
\hline 44 & 1-butanethiol & $\mathrm{C}_{4} \mathrm{H}_{10} \mathrm{~S}$ & 90.19 & Skunk & & 0.003 & 0.0002 & $<0.01$ \\
\hline 45 & $\begin{array}{l}\text { Ethyl methyl } \\
\text { sulfide }\end{array}$ & $\mathrm{C}_{3} \mathrm{H}_{8} \mathrm{~S}$ & 76.16 & $\begin{array}{l}\text { Sulfurous } \\
\text { odour }\end{array}$ & & 0.042 & 0.002 & $<0.01$ \\
\hline 46 & $\begin{array}{c}1,7,7- \\
\text { trimethyl- } \\
\text { bicyclo[2,2,1] } \\
\text {-heptan-2-one }\end{array}$ & $\mathrm{C}_{10} \mathrm{H}_{16} \mathrm{O}$ & $\begin{array}{c}152.2 \\
4\end{array}$ & $\begin{array}{c}\text { Fragrant } \\
\text { and } \\
\text { penetrating }\end{array}$ & & 2.84 & 0.1109 & $<0.01$ \\
\hline
\end{tabular}




\begin{tabular}{cccccccc}
\hline 47 & $\begin{array}{c}\text { 1,2-dichloro- } \\
\text { ethene }\end{array}$ & $\mathrm{C}_{2} \mathrm{H}_{4} \mathrm{Cl}_{2}$ & 96.94 & $\begin{array}{c}\text { Acrid, } \\
\text { chloroform } \\
\text {-like }\end{array}$ & 25 & 0.1437 & $<0.01$ \\
\hline 48 & $\begin{array}{c}1,1,1- \\
\text { trichloro- } \\
\text { ethene }\end{array}$ & $\mathrm{C}_{2} \mathrm{H}_{3} \mathrm{Cl}_{3}$ & $\begin{array}{c}133.4 \\
0\end{array}$ & $\begin{array}{c}\text { Sweet, } \\
\text { chlroform- } \\
\text { like }\end{array}$ & 5.3 & 0.0264 & $<0.01$ \\
\hline 49 & $\begin{array}{c}2,5 \text {-dimethyl- } \\
\text { furan }\end{array}$ & $\mathrm{C}_{6} \mathrm{H}_{8} \mathrm{O}$ & 96.13 & $\begin{array}{c}\text { Aromatic } \\
\text { caustic } \\
\text { odour }\end{array}$ & 100 & 0.0647 & $<0.01$ \\
\hline
\end{tabular}

Table 4 Odorants from the leachate using flux hood sampling

\begin{tabular}{|c|c|c|c|c|c|c|c|}
\hline Rank & Compound & Formula & MW & $\begin{array}{c}\text { Odour } \\
\text { Descriptor }\end{array}$ & $\begin{array}{c}\text { OT } \\
\text { ppm }^{[45]}\end{array}$ & $\begin{array}{c}\text { Concentration } \\
\left(\mathbf{m g} / \mathbf{m}^{3}\right)\end{array}$ & $\begin{array}{c}\text { Odour } \\
\text { Contribution } \\
(\%)\end{array}$ \\
\hline 1 & $\begin{array}{l}\text { Hydrogen } \\
\text { sulfide }\end{array}$ & $\mathrm{H}_{2} \mathrm{~S}$ & 34.08 & Rotten egg & 0.00041 & 0.0006 & 94.56 \\
\hline 2 & $\begin{array}{l}\text { Carbonyl } \\
\text { sulfide }\end{array}$ & COS & 60.08 & $\begin{array}{l}\text { Distinct sulfide } \\
\text { smell }\end{array}$ & 0.055 & 0.0031 & 2.06 \\
\hline 3 & $m$-xylene & $\mathrm{C}_{8} \mathrm{H}_{10}$ & 106.17 & Sweet, aromatic & 0.041 & 0.004 & 2.04 \\
\hline 4 & $p$-xylene & $\mathrm{C}_{8} \mathrm{H}_{10}$ & 106.17 & Sweet & 0.058 & 0.0016 & 0.58 \\
\hline 5 & $\begin{array}{l}1,2,4- \\
\text { trimetyl } \\
\text { benzene }\end{array}$ & $\mathrm{C}_{9} \mathrm{H}_{12}$ & 120.19 & Aromatic & 0.12 & 0.0021 & 0.31 \\
\hline 6 & Ethylbenzene & $\mathrm{C}_{8} \mathrm{H}_{10}$ & 106.17 & $\begin{array}{l}\text { Aromatic, } \\
\text { gasoline type }\end{array}$ & 0.17 & 0.0018 & 0.22 \\
\hline 7 & Toluene & $\mathrm{C}_{7} \mathrm{H}_{8}$ & 92.14 & Sweet, pungent & 0.33 & 0.0031 & 0.22 \\
\hline 8 & $\begin{array}{l}\text { Tetra- } \\
\text { hydrofuran }\end{array}$ & $\mathrm{C}_{4} \mathrm{H}_{8} \mathrm{O}$ & 72.11 & $\begin{array}{l}\text { Similar to } \\
\text { acetone }\end{array}$ & $30^{[47]}$ & 0.0053 & 0.01 \\
\hline
\end{tabular}

\subsection{Contributions of key odorants}

49 odorants from emission gas samples collected from landfill leachate collection pipe and 8 odorants from flux hood emission samples of the collected leachate sample were identified. Their contributions to the overall odour were determined based on their odour unit factors calculated from their concentrations and odour thresholds. The top 10 odorants are methyl mercaptan, ethyl mercaptan, m-xylene, $\mathrm{H}_{2} \mathrm{~S}, \mathrm{CS}_{2}, 1$,2,3,4-tetra-methylbenzene, pxylene, 1,2,4-trimethylbenzene, ethylbenzene and $\alpha$-pinene, which contributed more than $95 \%$ odour in the gas accumulated in the leachate collection pipe.

It may also need to remember that the calculation here was carried out on the basis of these assumption and facts:

- The key odorants investigated are only these compounds and contributions from other compounds are ignorable;

- The contributions of these odorants are additive to overall odour. Possibility of synergic or antagonistic as [54] found between these odorous compounds did not take into account because it is really hard to determine if the same function still exist when so many of different odorous compounds combine together.

- Available odour thresholds (even from different references) of these compounds are trustable and comparable. In order to avoid possible inconsistency and ensure reliability, most of OT values (Table 3 and Table 4) were quoted from [45] as they were obtained using the same triangular bag method. 


\subsection{Sampling and analytical method assessment based on the investigation results}

For VOCs sampling and analysis, significant (>10\%) breakthrough of the analytes in the second sampling tube was found despite a relatively low sample volume being collected $(1 \mathrm{~L})$. Figure 1 illustrates a pair of series sorbent tubes; the upper trace representing the front (first exposed to the sample stream) and the lower trace representing the back (second tube in series) tube. Both chromatograms are displayed on identical axes; despite the apparently low concentrations in the back (second tube in series) tube, the magnitude is significant. This tells us that more sampling options should be considered and managed properly when sampling from an unknown source in case of under-estimation or over-estimation of investigation sources.

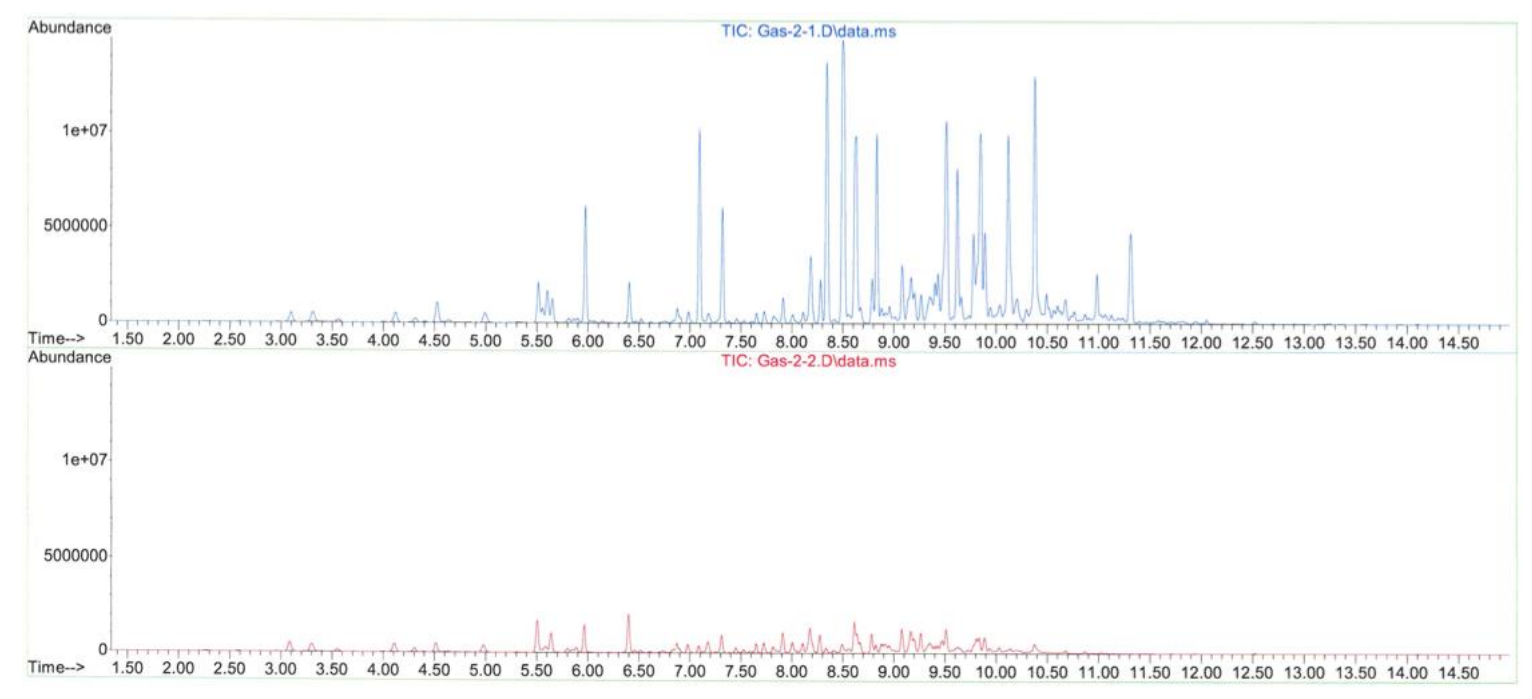

Figure 1 Total ion chromatograms from gas phase field samples

The results of the chemical speciation that have been performed on the gas samples collected on-site and the emission from the leachate collected indicate that the VOC and VSC emissions associated with the fugitive landfill gas were significantly greater than those from the leachate. This may also indicate that emissions from leachate are much complicate and different sampling and analytical method may give quiet different results.

As the differences between the Table 3 and Table 4 shows, different sampling and analytical methods may find quite different emission results. This also indicates that more sampling and measurement techniques are always better to be employed for finding all possible pollutants from an unknown pollution source.

\section{Conclusions}

Over 130 VOCs and VSCs were detected from the leachate emission of a landfill site in Sydney, Australia. 49 of them from landfill leachate collection pipe and 8 of them from flux hood emission were found to be odorous. The contributions of these odorants to overall odour emissions were also calculated based on their concentrations and odour thresholds. The top 10 odorants from leachate transportation pipe include methyl mercaptan, ethyl mercaptan, m-xylene, $\mathrm{H}_{2} \mathrm{~S}, \mathrm{CS}_{2}, 1,2,3$,4-tetra-methylbenzene, $\mathrm{p}$-xylene, 1,2,4trimethylbenzene, ethylbenzene and $\alpha$-pinene. They contributed more than $95 \%$ of the odour in the gas accumulated in the leachate collection pipe. Different results of different sampling and analytical methods indicates that more sampling and measurement techniques are always better for determination of the all possible pollutants from an unknown pollution source.

\section{References}

1. Kim, K.-H. Emission of reduced sulfur compounds (RSC) as a landfill gas (LFG): a comparative study of young and old landfill facilities. Atmos. Environ., 2006, 40, 6567-6578.

2. Song, S.-K.; Shon, Z.-H.; Kim, K.-H.; Kim, S. C.; Kim, Y.-K.; Kim, J.-K. Monitoring of atmospheric reduced sulfur compounds and their oxidation in two coastal landfill areas. Atmos. Environ., 2007, 41, 974-998.

3. Lee, S.; Xu, Q.; Booth, M.; Townsend, T. G.; Chadik, P.; Bitton, G. Reduced sulfur compounds in gas from construction and demolition debris landfills. Waste Manag., 2006, 26, 526-533.

4. Yue, D.; Han, B.; Sun, Y.; Yang, T. Sulfide emissions from different areas of a municipal solid waste landfill in China. Waste Manag., 2014, 34, 1041-1044.

5. Dincer, F.; Odabasi, M.; Muezzinoglu, A. Chemical characterization of odorous gases at a landfill site by gas chromatography-mass spectrometry. J. Chromatogr. A, 2006, 1122, 222-239. 
6.

Tassi, F., Montegrossi, G., Vaselli, O. and Liccioli, C. Degradation of C2-C15 volatile organic compounds in a landfill cover soil. Sci. Total Environ., 2009, 407, 4513-4525.

7. Capelli, L.; Sironi, S.; Del Rosso, R.; Céntola, P.; Il Grande, M. A comparative and critical evaluation of odour assessment methods on a landfill site. Atmos. Environ., 2008, 42, 7050-7058.

8. Scaglia, B.; Orzi, V.; Artola, A.; Font, X.; Davoli, E.; Sanchez, A. Adani, F. Odours and volatile organic compounds emitted from municipal solid waste at different stage of decomposition and relationship with biological stability. Bioresour. Technol., 2011, 102, 4638-4645.

9. Lehtinen, J.; Tolvanen, O.; Nivukoski, U.; Veijanen, A.; Hänninen, K. Occupational hygiene in terms of volatile organic compounds (VOCs) and bioaerosols at two solid waste management plants in Finland. Waste Manag., 2013, 33, 964-973.

10. Duan, Z.; Lu, W.; Li, D.; Wang, H. Temporal variation of trace compound emission on the working surface of a landfill in Beijing, China. Atmos. Environ., 2014., 88, 230-238.

11. Zou, S. C.; Lee, S. C.; Chan, C. Y.; Ho, K. F.; Wang, X. M.; Chan, L. Y.; Zhang, Z. X. Characterization of ambient volatile organic compounds at a landfill site in Guangzhou, South China. Chemosphere, 2003, 51, 10151022.

12. Chiriac, R.; Carre, J.; Perrodin, Y.; Fine, L.; Letoffe, J.-M. Characterisation of VOCs emitted by open cells receiving municipal solid waste. J. Hazard. Mater., 2007, 149, 249-263.

13. Gallego, E.; Perales, J. F.; Roca, F. J.; Guardino, X. Surface emission determination of volatile organic compounds (VOC) from a closed industrial waste landfill using a self-designed static flux chamber. Sci. Total Environ., 2014, 470-471, 587-599.

14. Majumdar, D.; Srivastava, A. Volatile organic compound emissions from municipal solid waste disposal sites: a case study of Mumbai, India. J. Air Waste Manag. Assoc., 2012, 62(4), 398-407.

15. Eklund, B.; Anderson, E. P.; Waler, B. L.; Burrows, D. B. Characterization of landfill gas composition at the Fresh Kills municipal solid-waste landfill. Environ. Sci. Technol., 1998, 32, 2233-2237.

16. Schweigkofler, M.; Niessner, R. Determination of siloxane and VOC in landfill gas and sewage gas by canister sampling and GC-MS/AES analysis. Environ Sci.Technol., 1999, 33, 3680-3685.

17. Young, P. J.; Parker, A. The identification and possible environmental impact of trace gases and vapours in landfill gas. Waste Manag. Res., 1983, 1, 213-226.

18. Loizidou, M.; Kapetanios, E. G. Study on the gaseous emissions from a landfill. Sci. Total Environ., 1992, 127, 201210.

19. Ding, Y.; Cai, C.; Hu, B.; Xu, Y.; Zheng, X.; Chen, Y.; Wu W. Characterization and control of odorous gases at a landfill site: a case study in Hangzhou, China. Waste Manag., 2012, 32, 317-326.

20. Ray, S.; Kim, K.-H.; Yoon, H.-O. Effect of incineration on the removal of key offensive odorants released from a landfill leachate treatment station. Chemosphere, 2012, 87, 557-565.

21. Chiriac, R.; Carré, J.; Perrodin, Y.; Vaillant, H.; Gasso, S.; Miele, P. Study of the dispersion of VOCs emitted by a municipal solid waste landfill. Atmos. Environ., 2009, 43(11), 1926-1931.

22. Vilavert, L.; Nadal, M.; Figueras, M. J.; Domingo, J. L.Volatile organic compounds and bioaerosols in the vicinity of a municipal waste organic fraction treatment plant, human health risks. Environ. Sci. Pollut. Res., 2012, 19(1), 96-104.

23. Romain, A.-C.; Delva, J.; Nicolaas, J. Complementary approaches to measure environmental odours emitted by landfill areas. Sens. Actuators. B Chem., 2008, 131, 18-23.

24. Junyapoon, Sl; Bartle, K.; Ross, A. B.; Cooke, M. Analysis of malodorous sulfur gases and volatile organometalloid compounds in landfill gas emissions using capillary gas chromatography with programmed temperature vaporization injection and atomic emission detection. Int. J. Environ. Anal. Chem., 2002, 82(2), 47-59.

25. Allen, M. R.; Brithwaite, A.; Hills, C. C. Trace organic compounds in landfill gas at seven UK waste disposal sites. Environ. Sci. Technol., 1997. 31 (4), 1054-1061.

26. Paxéus, N. Organic compounds in municipal landfill leachates. Water Sci.Technol., 2000, 42, 323-333.

27. Rodríguez-Navas, C.; Forteza, R.; Cerdà, V. Use of thermal desorption-gas chromatography-mass spectrometry (TD-GC-MS) on identification of odorant emission focus by volatile organic compound characterisation. Chemosphere, 2012, 89, 1426-1436.

28. Kim, K.-H.; Choi, YJ; Jeon, EC; Sunwoo, Y. Characterization of malodorous sulfur compounds in landfill gas. Atmospheric Environment, 2005, 39, 1103-1112.

29. Shon, Z.-H.; Kim, K.-H.; Jeon, E.-C.; Kim, M.-Y.; Kim, Y.-K.; Song, S.-K. Photochemistry of reduced sulfur compounds in a landfill environment. Atmos. Environ., 2005, 39, 4803-4814.

30. Fang, J.-J.; Yang, N.; Cen, D.-Y.; Shao, L.-M.; He, P.-J. Odor compounds from different sources of landfill: characterization and source identification. Waste Manag., 2012, 32, 1401-1410.

31. Xu, Q.; Townsend, T. Factors affecting temporal H2S emission at construction and demolition (C\&D) debris landfills. Chemosphere, 2014, 96, 105-111.

32. Davoli, E.; Gangai, M. L.; Morselli, L; Tonelli, D. Characterisation of odorants emissions from landfills by SPME and GC/MS. Chemosphere, 2003, 51, 357-368.

33. Palmiotto, M.; Fattore, E.; Paiano, V.; Celeste, G.; Colombo, A.; Davoli, E. Influence of a municipal solid waste landfill in the surrounding environment: toxicological risk and odor nuisance effects. Environ. Int., 2014, 68, 16-24.

34. Bruno, P.; Caselli, M.; de Gennaro, G.; Solito, M.; Tutino, M. Monitoring of odor compounds produced by solid waste treatment plants with diffusive samplers. Waste Management, 2007, 27, 539-544.

35. Sadowska-Rociek, A.; Kurdziel, M.; Riesenmey, P., K.; Vaillant, H.; Batton-Hubert, M.; Szczepaniec-Cięciak, E. Analysis of odorous compounds at municipal landfill sites. Waste Manag. \& Res., 2009, 00, 1-10. 
36. Schwarzbauer, J.; Heim, S.; brinker, S.; Littke, R. Occurrence and alteration of organic contaminants in seepage and leakage water from a waste disposal landfill. Water Res., 2002, 36, 2275-2287.

37. Muezzinoglu, A. A study of volatile organic sulfur emissions causing urban odors. Chemosphere, 2003, 51, 245252.

38. Benfenati, E.; Pierucci, P.; Fanelli, R.; Preiss, A.; Godejohann, M.; Astratov, M.; Levsen, K.; Barceló, D. Comparative studies of the leachate of an industrial landfill by gas chromatography-mass spectrometry, liquid chromatography-nuclear magnetic resonance and liquid chromatography-mass spectrometry. J. Chromatogr. A, 1999, 831, 243-256.

39. Öman, C; Junestedt, C. Chemical characterization of landfill leachates - 400 parameters and compounds. Waste Manag., 2008, 28, 1876-1891.

40. Turki, N.; Belhaj, D.; Jaabiri, I.; Ayadi, H.; Kallel, M.; Bouzid, J. Determination of organic compounds in landfill leachates treated by coagulation-flocculation and fenton-adsorption. JESTFT, 2013, 7(3), 18-25.

41. Öman, C.; Hynning, P.-Å. Identification of organic compounds in municipal landfill leachates. Environ. Pollut., 1993, 80, 265-271.

42. Hurst, C.; Longhurst, P.; Pollard, S.; Smith, R.; Jefferson, B.; Gronow, J. Assessment of municipal waste compost as a daily cover material for odour control at landfill sites. Environ. Pollut., 2005, 135, 171-177.

43. Chen, S.-J.; Hsieh, L.-T.; Hwang, W.-I.; Xu, H.-C.; Kao, J.-H. Abatement of odor emissions from landfills using natural effective microorganism enzyme. Aerosol Air Qual. Res., 2003, 3(1), 87-99.

44. Brosseau, J.; Heitz, M. Trace gas compound emissions from municipal landfill sanitary sites. Atmos. Enviro.t, 1994 , 28(2), 285-293.

45. Nagata Yoshio. Measurement of odor threshold by triangle odor bag method. Odour Measurement Review, Japan Ministry of the Environment. 2003, 118-127.

46. van Gemert, L. J., 2003. Odour Thresholds. Published by Oliemans Punter \& Partners BV, The Netherlands.

47. Summer, W. 1975. Odour Pollution of Air: Cause and Control. L. Hill, London.

48. US EPA, Measurement of gases emission rates from land surfaces using an emission isolation flux chamber - user's guide, EPA 600/8-86-008 (NTIS PB86-223161), February 1986.

49. Wang, X.; Jiang J.; Kaye, R. Improvement of a wind-tunnel sampling system for odour and VOCs. Water Sci. Technol., 2001, 44(9), 71-77.

50. Australian and New Zealand standard, 2009. Stationary source emissions - Method 4: Area source sampling-Flux chamber technique (AS/NZS 4323.4:2009).

51. Department of Environment and Conservation (NSW), Approved Methods for the sampling and analysis of air pollutants in New South Wales. August 2005.

52. Wang, X., Parcsi, G., Sivret, E., Le, H., Wang, B. \& Stuetz, R. M. Odour emission ability (OEA) and its application in assessing odour removal efficiency. Water Sci. Technol., 66(9), 1828-1833.

53. Firor, R. L. and Quimby, B. D., A Comparison of Sulfur Selective Detectors for Low Level Analysis in Gaseous Streams Application. Available online: https://www.agilent.com/cs/library/applications/5988-2426EN\%20.pdf (accessed on 21 November 2019).

54. Jarauta, I.; Ferreira, V.; Cacho, J. F. Synergic, additive and antagonistic effects between odorants with similar odour properties. Dev. Food Sci., 2006, 43, 205-208. 